\title{
Description of the JMA Operational Spectral Model
}

\author{
By M. Kanamitsu, K. Tada, T. Kudo, N. Sato and S. Isa \\ Electronic Computation Center, Japan Meteorological Agency, Tokyo 100, Japan \\ (Manuscript received 26 August 1983, in revised form 14 October 1983)
}

\begin{abstract}
A 12-level hemispheric spectral model has been developed for operational purpose. The model has a horizontal resolution of triangular 42 truncation and incorporates full physical processes including diurnal variation of shortwave radiation and cloud effects.

The model has been tested for nearly a year before introduction as an operational model, and compared with the grid point model which was operational at that time. It has been shown that the spectral model was superior to the grid point model in many respects. The reason is due to various factors, i.e., higher resolution, initialization, analysis etc., and not just due to the use of the spectral method.

The useful predictability of the spectral model is found to be 5-6 days. The model forecast is quite useful in predicting sudden developments of baroclinic disturbances, typhoon movements and other important meteorological phenomena with reasonable accuracy.
\end{abstract}

\section{Introduction}

The accuracy of the numerical weather prediction (NWP) has increased considerably in recent years. Major factors contributing to the improvement of NWP are the developments in computer technology and in the theoretical and observational meteorology. In the theoretical meteorology, for example, extension of optimum interpolation method for analysis (e.g. Lorenc, 1981), nonlinear normal mode initialization (Machenhauer, 1977) and transform method for the integration of spectral form of equations (Orszag, 1970) are considered to be the most important recent developments in the NWP and analysis technologies.

European Centre for Medium Range Weather Forecasts (ECMWF) is the first that has produced considerably accurate forecast combining above recently developed technologies with high resolution global forecast model (Hollingsworth et al., 1980). Encouraged by the ECMWF's success, other meteorological centers have been improving their NWP models quite rapidly. In the recent WMO publication on the international comparison project of the NWP models (Bengtsson and Lange, 1982), we can clearly see the drastic improvements of NWPs in the meteorological centers over the world.
With these recent developments, predictability of NWP models seems to have reached another limit. In order to break through this new limit of predictability, study on the forecast errors in NWP models have been emphasized. In fact, this theme is regarded as one of the most important problems in the field of NWP (ECMWF Seminar, 1981).

At the Japan Meteorological Agency (JMA), a new model has been introduced as an operational model in March 1983. This model also incorporates the recent developments in NWP technologies. Various statistics of the forecasts collected during the test period have indicated that the model has reached satisfactory level of forecast accuracy.

The present paper describes dynamical and physical framework of the forecast model, its capability, namely its objective forecast scores and some synoptic examples of the forecasts.

The forecast model described in this paper is not particularly original in any sense. However, we have used major space in this paper to describe the model. The objective is to present the degree of sophistication of the forecast model that is required to produce the forecast of the quality discussed in the later sections. Furthermore, this paper is designed to form introduction of the papers to follow, that deal 
with the main theme of the forecast error. Since forecast error depends strongly on the dynamics and physics of the forecast model, initialization and analysis method, somewhat detailed description of the model is necessary.

\section{Forecast model}

The new operational model at JMA is a 12level northern hemispheric spectral model. The major roles of the model in the operational analysis forecast system are (1) to perform 3 to $\delta$ day forecast of the large scale flow, (2) to provide time varying boundary conditions for the fine mesh limited area model and (3) to provide initial guess for the analysis. The basic framework of the spectral model is based on Bourke (1974) and Hoskins and Simmons (1975).

\section{(1) Structure of the model}

(a) Vertical resolution

The model has 12 levels in the vertical. The arrangement of the sigma levels is shown in Fig. 1. The variables $\phi, \chi, T, \phi$, and $q$ (see Table 1 for the meaning of the variables) are placed at the same level and $\dot{\sigma}$ at the staggered level. The boundary condition of $\dot{\sigma}=0$ are applied at $\sigma=1$ and 0 . In order to increase accuracy of forecast at the surface, vertical resolution is increased in the planetary boundary layer (below $800 \mathrm{hPa}$ ).

(b) The dependent variables are expanded in terms of the spherical harmonics using the triangular wave number truncation at wave number 42. The Gaussian grid of 128 grid points in east-west and 32 grid points between the north pole and the equator are used for the computations of nonlinear terms and the physical processes.

\section{(2) Prediction equations}

The spectral prediction equations are written in the following compact forms using matrix notations introduced by Hoskins and Simmons (1975). The basic equation in the physical space are found in Appendix I. We introduce here a column matrix (-) with $N$ elements ( $N$ being the number of the model sigma levels), $N \times N$ square matrix $(=)$ and a row matrix $(-)^{T}$. An example of the column matrix is shown as:

$$
\underline{\Phi}_{n}{ }^{m}=\left(\begin{array}{c}
\Phi_{n}{ }^{m}{ }_{k=1} \\
\Phi_{n}{ }^{m}{ }_{k=2} \\
\vdots \\
\Phi_{n}{ }^{m}{ }_{k=N}
\end{array}\right)
$$

Vorticity equation

$$
-n(n+1) \underline{\dot{\phi}}_{n}{ }^{m}=\underline{E}_{n}{ }^{m}+\left(\mathscr{D}_{\xi}\right)_{n}{ }^{m}
$$

Divergence equation

$$
\begin{aligned}
- & n(n+1) \underline{\dot{x}}_{n}{ }^{m} \\
= & \underline{F}_{n}{ }^{m}+\frac{n(n+1)}{a^{2}}\left(\underline{\Phi}_{n}{ }^{m}+R \bar{T} \pi_{n}{ }^{m}\right)+(\underline{\mathscr{D}}) n^{m}
\end{aligned}
$$

Thermodynamic equation

$$
\begin{aligned}
\underline{\dot{T}}_{n}{ }^{m}= & \underline{H}_{n}{ }^{m}-J_{n}{ }^{m}+\left(\underline{\mathscr{D}}_{H}\right)_{n}{ }^{m} \\
= & \underline{H}_{n}{ }^{m} \\
& +\frac{n(n+1)}{a^{2}}\left\{\underline{\underline{Q}} \underline{\underline{S}}+\frac{R \bar{T}}{C_{p}} \underline{C}^{T}\right\} \underline{\chi}_{n}{ }^{m} \\
& \left.\quad+(\underline{\mathscr{D}})_{n}\right)^{m}
\end{aligned}
$$

Moisture equation

$$
\underline{\dot{q}}_{n}{ }^{m}=\underline{M}_{n}{ }^{m}+\left(\underline{\mathscr{D}_{q}}\right)_{n}{ }^{m}
$$

Continuity equation, tendency equation and equation for $\dot{\sigma}$

$$
\dot{\pi}_{n}^{m}=\left(\frac{\partial \pi_{D}}{\partial t}\right)_{n}^{m}+Z_{n}^{m}
$$

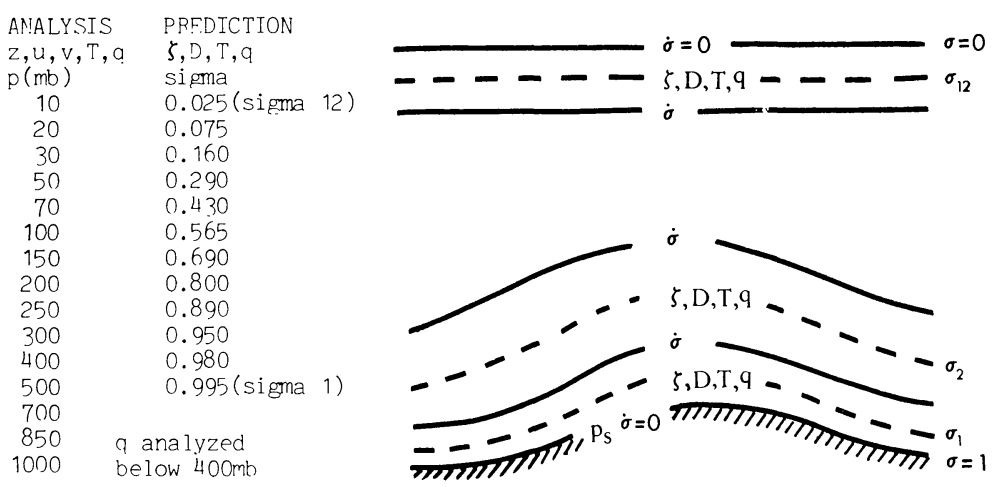

Fig. 1 Model sigma levels and arrangement of variables. Analyses levels are also indicated at the left side. 
Table 1. List of symbols

$a:$ Earth's radius

$C_{p}$ : Specific heat of dry air at constant pressure

$D$ : Divergence

$F_{H}$ : Vertical eddy flux of heat

$F_{q}$ : Vertical eddy flux of moisture

$F_{v}$ : Vertical eddy flux of momentum

$f:$ Coriolis parameter

$g$ : Accerelation of gravity

$i$ : Unit imaginary number

$m:$ Zonal wavenumber index

$n$ : Meridional node index

$P$ : Precipitation

$p$ : Pressure

$p_{s}:$ Surface pressure

$P_{n}{ }^{m}$ : Associated Legendre functions of the order $n, m$

$Q$ : Diabatic heating rate

$q:$ Specific humidity

$R:$ Gas constant of dry air

$T:$ Temperature

$t:$ Time

$u$ : East-west component of wind

$U:=u \cdot \cos \phi$

$v$ : North-sourth component of wind

$V:=v \cdot \cos \phi$

$\zeta:$ Vorticity

$\kappa:=R / C_{p}$

$\lambda$ : Longitude

$\mu:=\sin \phi$

$\pi:=\ln p_{\mathrm{s}}$

$\rho:$ Density of air

$\sigma:=p / p_{s}$

$\tau:$ Stress tensor

$\chi:$ Velocity potential

$\psi:$ Stream function

$\Phi$ : Geopotential

$\phi:$ Latitude

$\Omega$ : Earth's rotation rate

$\nabla^{2}:$ Laplacian operator

$$
\begin{aligned}
& \left(\frac{\partial \pi_{D}}{\partial t}\right)_{n}^{m}=\frac{n(n+1)}{a^{2}} \underline{C}^{T} \underline{\chi}_{n}{ }^{m} \\
& \left(\dot{\sigma}_{D}\right)_{n}{ }^{m}=\frac{n(n+1)}{a^{2}} \underline{S}_{\underline{\chi}} \underline{n}^{m}
\end{aligned}
$$

Hydrostatic equation

$$
\underline{\Phi}_{n}^{m}-\left(\underline{\Phi}_{s}\right)_{n}{ }^{m}=\underline{\underline{W}} \underline{T}_{n}{ }^{m}
$$

The elements of the column matrices of the nonlinear terms $E_{n}{ }^{m}, F_{n}{ }^{m}, H_{n}{ }^{m}, J_{n}^{m}, M_{n}{ }^{m}$, and $Z_{n}{ }^{m}$ are expressed as follows.

$$
\begin{aligned}
& E_{n}^{m}=-\frac{1}{2 \pi} \int_{0}^{1} \int_{0}^{2 \pi} \frac{1}{a \cos ^{2} \phi}\left(i m A P_{n}{ }^{m}\right. \\
& \left.-B \cos \phi \frac{d P_{n}^{m}}{d \phi}\right) e^{-i m \lambda} d \lambda d \mu \\
& F_{n}{ }^{m}=\frac{1}{2 \pi} \int_{0}^{1} \int_{0}^{2 \pi} \frac{1}{a \cos ^{2} \phi}\left(\operatorname{im} B P_{n}{ }^{m}\right. \\
& \left.+A \cos \phi \frac{d P_{n}^{m}}{d \phi}\right) e^{-\imath m \lambda} d \lambda d \mu \\
& +\frac{n(n+1)}{a^{2}} \frac{1}{2 \pi} \int_{0}^{1} \int_{0}^{2 \pi}\left(\frac{U^{2}+V^{2}}{2 \cos ^{2} \phi}\right) \\
& \text { - } \boldsymbol{P}_{n}{ }^{m} e^{-i m \lambda} d \lambda d \mu \\
& H_{n}{ }^{m}=-\frac{1}{2 \pi} \int_{0}^{1} \int_{0}^{2 \pi} \frac{1}{a \cos ^{2} \phi}\left(i m U T^{\prime} P_{n}{ }^{m}\right. \\
& \left.-V T^{\prime} \cos \phi \frac{d P_{n}^{m}}{d \phi}\right) e^{-i m \lambda} d \lambda d \mu \\
& +\frac{1}{2 \pi} \int_{0}^{1} \int_{0}^{2 \pi} L P_{n}^{m} e^{-i m \lambda} d \lambda d \mu \\
& J_{n}^{m}=\left(\dot{\sigma}_{D}\right)_{n}{ }^{m} \sigma^{\kappa} \bar{T} \frac{\partial \sigma^{-\kappa}}{\partial \sigma}+\frac{R \bar{T}}{C_{p}}\left(\frac{\partial \pi_{D}}{\partial t}\right)_{n}^{m} \\
& M_{n}{ }^{m}=-\frac{1}{2 \pi} \int_{0}^{1} \int_{0}^{2 \pi} \frac{1}{a \cos ^{2} \phi}\left(i m U q P_{n}{ }^{m}\right. \\
& \left.-V q \cos \phi \frac{d P_{n}^{m}}{d \phi}\right) e^{-i m \lambda} d \lambda d \mu \\
& +\frac{1}{2 \pi} \int_{0}^{1} \int_{0}^{2 \pi} N P_{n}^{m} e^{-i m \lambda} d \lambda d \mu \\
& Z_{n}{ }^{m}=\frac{1}{2 \pi} \int_{0}^{1} \int_{0}^{2 \pi} Z P_{n}^{m} e^{-i m \lambda} d \lambda d \mu
\end{aligned}
$$

where the wave to physical space conversion and terms defined on the Gaussian grids are expressed as follows.

$$
\begin{aligned}
& (T, \Phi, q, \pi) \\
& =\sum_{m=-42}^{42} \sum_{n=|m|}^{42}\left(T_{n}^{m}, \Phi_{n}^{m}, q_{n}^{m}, \pi_{n}^{m}\right) P_{n}{ }^{m} e^{i m \lambda} \\
& (\zeta, D)=\sum_{m=-42}^{42} \sum_{n=|m|}^{42} \\
& -n(n+1)\left(\psi_{n}^{m}, \chi_{n}{ }^{m}\right) \boldsymbol{P}_{n}{ }^{m} e^{i m \lambda} \\
& U=a \sum_{m=-42}^{42} \sum_{n=|m|}^{42}\left(-\phi_{n}{ }^{m} \cos \phi \frac{d P_{n}{ }^{m}}{d \phi}\right. \\
& \left.+i m \chi_{n}^{m} \boldsymbol{P}_{n}^{m}\right) e^{i m \lambda} \\
& V=a \sum_{m=-42}^{42} \sum_{n=|m|}^{42}\left(\chi_{n}^{m} \cos \phi \frac{d P_{n}^{m}}{d \phi}\right. \\
& \left.+i m \psi_{n}^{m} \boldsymbol{P}_{n}^{m}\right) e^{i m \lambda}
\end{aligned}
$$


$\pi=\ln p_{s}$

$T^{\prime}=T-\bar{T}$

$\bar{T}=$ constant $=300^{\circ} \mathrm{K}$

$\vec{V} \cdot \nabla \pi=\frac{1}{a \cos ^{2} \phi}\left(U \frac{\partial \pi}{\partial \lambda}+V \cos \phi \frac{\partial \pi}{\partial \phi}\right)$

$\frac{\partial \pi}{\partial \lambda}=\sum_{m=-42}^{42} \sum_{n=|m|}^{42} i m \lambda P_{n} m e^{i m \lambda}$

$\cos \phi \frac{\partial \pi}{\partial \phi}=\sum_{m=-42}^{42} \sum_{n=|m|}^{42}\left(-\pi_{n}^{m} \cos \phi \frac{d P_{n}^{m}}{e \phi}\right)$ $\cdot e^{i m \lambda}$

$\frac{\partial \pi_{D}}{\partial t}=-\int_{0}^{1} D d \sigma$

$\frac{\partial \pi_{A}}{\partial t}=-\int_{0}^{1} \vec{V} \cdot \nabla \pi d \sigma=Z$

$\dot{\sigma}=\dot{\sigma}_{A}+\dot{\sigma}_{D}$

$\left(\dot{\sigma}_{D}\right)_{\sigma+\Delta \sigma}=\left(\dot{\sigma}_{D}\right)_{\sigma}-\int_{\sigma}^{\sigma+\Delta \sigma} D d \sigma+\Delta \sigma \int_{0}^{1} D d \sigma$

$\left(\dot{\sigma}_{A}\right)_{\sigma+\Delta \sigma}=\left(\dot{\sigma}_{A}\right)_{\sigma}$

$$
-\int_{\sigma}^{\sigma+\Delta \sigma} \vec{V} \cdot \nabla \pi d \sigma+\Delta \sigma \int_{0}^{1} \vec{V} \cdot \nabla \pi d \sigma
$$

$$
\begin{aligned}
A= & (\zeta+f)) U+\dot{\sigma} \frac{\partial V}{\partial \sigma}+\frac{R T^{\prime}}{a} \cos \phi \frac{\partial \pi}{\partial \phi} \\
& +\cos \phi \frac{g}{p_{s}} \frac{\partial \tau_{\phi}}{\partial \sigma}
\end{aligned}
$$

$B=(\zeta+f) V-\dot{\sigma} \frac{\partial U}{\partial \sigma}-\frac{R T^{\prime}}{a} \frac{\partial \pi}{\partial \lambda}$

$-\cos \phi \frac{g}{p_{s}} \frac{\partial \tau_{\lambda}}{\partial \sigma}$

$$
\begin{aligned}
L=- & \dot{\sigma}_{A} \sigma^{\kappa} \frac{\partial}{\partial \sigma} T \sigma^{-\kappa}-\dot{\sigma}_{D} \sigma^{\kappa} \frac{\partial}{\partial \sigma} T^{\prime} D \\
& +\frac{R T^{\prime}}{C_{p}} \frac{\partial \pi_{D}}{\partial t}+\frac{R T}{C_{p}}\left(\frac{\partial \pi_{A}}{\partial t}+\vec{V} \cdot \nabla \pi\right) \\
& +\frac{Q}{C_{p}}+\frac{1}{C_{p}} \frac{g}{p_{s}} \frac{\partial F_{H}}{\partial \sigma}
\end{aligned}
$$

$$
N=-\dot{\sigma} \frac{\partial q}{\partial \sigma}+q D-P+\frac{g}{p_{s}} \frac{\partial F_{q}}{\partial \sigma}
$$

It may be helpful to note here that the complex form of the vertical advection terms in the thermodynamic equation (first and the second terms on the right hand side of Eq. 33) is due to the conservation of potential temperature. Furthermore, the particular way of dividing the terms in the thermodynamic equation to $H_{n}{ }^{m}$ and $J_{n}{ }^{m}$ (terms linearly related to divergence) terms is to apply a semi-implicit time integration scheme.

The associated Legendre functions of the first kind $P_{n}{ }^{m}$ used in this paper are normalized to 2 and expressed as:

$$
\begin{aligned}
& P_{n}^{m}=\sqrt{(2 n+1) \frac{(n-m) !}{(n+m) !}} \cos ^{m} \phi \frac{d^{m} P_{n}(\mu)}{d \mu^{m}} \\
& P_{n}(\mu)=\frac{1}{2^{n} n !} \frac{d^{n}}{d \mu^{n}}\left(\mu^{2}-1\right)^{n} \\
& \mu=\sin \phi
\end{aligned}
$$

The matrices $\underline{\underline{Q}}, \underline{=}, \underline{C}^{T}$ and $\underline{\underline{W}}$ that appear in Eqs. $4^{\prime}, 7,8$ and 9 are determined from finite difference scheme used for vertical temperature advection, $\dot{\sigma}$ computation, vertical integration of divergence and hydrostatic equation, respectively, and take the following forms:

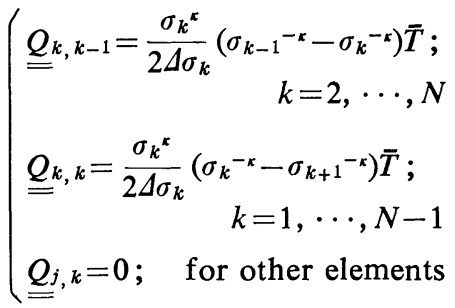

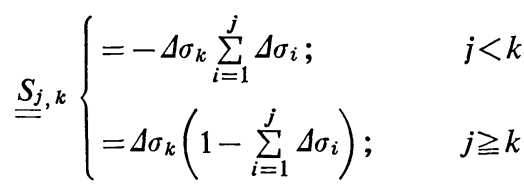

$$
\begin{aligned}
& \underline{C}_{k}^{T}=\Delta \sigma_{k}
\end{aligned}
$$

and

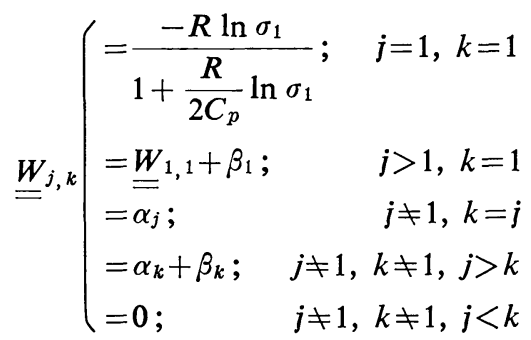

here,

$$
\begin{aligned}
& \alpha_{k}=\frac{C_{p}}{2}\left\{\left(\frac{\sigma_{k-1}}{\sigma_{k}}\right)^{\kappa}-1\right\} \\
& \beta_{k}=\frac{C_{p}}{2}\left\{1-\left(\frac{\sigma_{k+1}}{\sigma_{k}}\right)^{\kappa}\right\}
\end{aligned}
$$

The finite differencing scheme applied here (Arakawa, 1972) conserves potential temperature 
and its squared quantity. Furthermore, it guarantees the conservation of total energy.

Other terms that require finite difference in the vertical are the vertical advection of momentum and moisture, that appear in Eqs. 31, 32 and 34. For those terms, the finite difference form;

$$
\begin{aligned}
\dot{\boldsymbol{\sigma}} \frac{\partial f}{\partial \sigma}= & \frac{1}{2 \Delta \sigma}\left\{\dot{\boldsymbol{\sigma}}_{k-1 / 2}\left(f_{k-1}-f_{k}\right)\right. \\
& \left.+\dot{\boldsymbol{\sigma}}_{k+1 / 2}\left(f_{k}-f_{k+1}\right)\right\}
\end{aligned}
$$

is used, where $f$ is $U, V$ or $q$.

The vertical diffusion terms (last terms on the right hand side of Eqs. 31-34) are treated separately and will be discussed later.

For the numerical integration of Eqs. 10-15, Fast Fourier Transform (FFT) is used for the integral with respect to $\lambda$ and the Gaussian quadrature for $\phi$ (Eliasen et al., 1970).

\section{(3) Time differencing scheme}

For saving computational time, semi-implicit time integration scheme of the form described below (Hoskins and Simmons, 1975) is applied.

$$
\begin{aligned}
& -\frac{n(n+1)}{a^{2}} \delta_{t} \underline{\psi}_{n}{ }^{m}=\underline{E}_{n}{ }^{m} \\
& -\frac{n(n+1)}{a^{2}} \delta_{t} \underline{\chi}_{n}{ }^{m} \\
& \left.\quad=\underline{F}_{n}{ }^{m}+\frac{n(n+1)}{a^{2}}{\underline{\left(\Phi_{n}\right.}}^{m}+R \bar{T} \pi_{n}^{m}\right) \\
& \delta_{t} \underline{T}_{n}{ }^{m}=\underline{H}_{n}{ }^{m}+\frac{n(n+1)}{a^{2}}\left\{\underline{Q S}+\frac{R \bar{T}}{C_{p}} \underline{C}^{T}\right\}{\overline{\chi_{n}}}^{m} t
\end{aligned}
$$

$$
\delta_{t} \underline{q}_{n}^{m}=\underline{M}_{n}^{m}
$$

where

$$
\begin{aligned}
\delta_{t}(\quad) & =\frac{()_{t+\Delta t}-()_{t-\Delta t}}{2 \Delta t} \\
(-t & =\frac{()_{t+\Delta t}+()_{t-\Delta t}}{2}
\end{aligned}
$$

Time step $\Delta t$ is determined as a function of the maximum wind speed,

$$
\Delta t=\frac{a}{\left|V_{\max }\right| n}
$$

where $n$ is the truncation wavenumber $(n=42)$. $\Delta t$ is computed once in 24 hours (forecast time) and assuring computational stability, it is reduced to $80 \%$ of the calculated value.

Computational mode is controled by the time filter (Asselin, 1972) with the damping factor of 0.05 .
It is noted that the vertical diffusion terms included in the terms $\underline{E}_{n}{ }^{m}, \underline{F}_{n}{ }^{m}, \underline{H}_{n}{ }^{m}$ and $\underline{M}_{n}{ }^{m}$ are treated separately from the above time differencing scheme in order to prevent computational instability. (see (4)-b below)

\section{(4) Physical processes in the model}

(a) Horizontal diffusion

The major purpose of incorporating horizontal diffusion in the spectral model is to prevent noise accumulation due to the spectral blocking. The forms of the diffusion used in the model are (Orszag, 1974):

$$
\begin{aligned}
& \mathscr{D}_{\zeta}=K\left(\nabla^{4} \zeta+\frac{4 \zeta}{a^{4}}\right) \\
& \mathscr{D}_{D}=K\left(\nabla^{4} D+\frac{4 D}{a^{4}}\right) \\
& \mathscr{D}_{H}=K V^{4} T \\
& \mathscr{D}_{q}=K V^{4} q
\end{aligned}
$$

where $K$ is the horizontal diffusion coefficient.

The spectral form of these terms are

$$
\begin{aligned}
& \left(\mathscr{D}_{\zeta}\right)_{n}{ }^{m}=K \frac{n^{2}(n+1)^{2}-4}{a^{4}} \cdot \frac{n(n+1)}{a^{2}} \psi_{n}{ }^{m} \\
& \left(\mathscr{D}_{D}\right)_{n}{ }^{m}=K \frac{n^{2}(n+1)^{2}-4}{a^{4}} \cdot \frac{n(n+1)}{a^{2}} \chi_{n}{ }^{m} \\
& \left(\mathscr{D}_{H}\right)_{n}{ }^{m}=-K \frac{n^{2}(n+1)^{2}}{a^{4}} T_{n}{ }^{m} \\
& (\mathscr{D} q)_{n}{ }^{m}=-K \frac{n^{2}(n+1)^{2}}{a^{4}} q_{n}{ }^{m}
\end{aligned}
$$

$K$ is presently assumed to be $7.8 \times 10^{15} \mathrm{~m}^{4}$ $\sec ^{-1}$. In order to prevent numerical instability, forward time differencing scheme is applied to those terms.

(b) Vertical diffusion

Vertical fluxes of momentum, heat and moisture within the atmosphere are defined as

$$
\begin{aligned}
& \tau_{\phi}=-\frac{\rho^{2} g}{p_{s}} K_{V} \frac{\partial v}{\partial \sigma} \\
& \tau_{\lambda}=-\frac{\rho^{2} g}{p_{s}} K_{V} \frac{\partial u}{\partial \sigma} \\
& F_{H}=\frac{C_{p} \rho^{2} g}{p_{s}} K_{H} \frac{\partial \theta}{\partial \sigma} \\
& F_{q}=\frac{\rho^{2} g}{p_{s}} K_{q} \frac{\partial q}{\partial \sigma}
\end{aligned}
$$

and the surface fluxes as

$$
\begin{aligned}
& \tau_{\phi}=\rho_{0} C_{D}\left|\vec{V}_{0}\right| v_{0} \\
& \tau_{\lambda}=\rho_{0} C_{D}\left|\vec{V}_{0}\right| u_{0}
\end{aligned}
$$




$$
\begin{aligned}
& F_{H}=C_{p} \rho_{0} C_{H}\left|\vec{V}_{0}\right|\left(T_{\mathrm{S} F}-T_{0}\right) \\
& F_{q}=\rho_{0} C_{q}\left|\overrightarrow{\boldsymbol{V}}_{0}\right|\left(q_{\mathrm{SF}}-q_{0}\right)
\end{aligned}
$$

where $\rho$ stands for density of dry air. The subscript 0 denotes values at the level just above the surface and $S F$ denotes values of the earth's surface. $T_{0}$ is computed from extrapolation of $T_{k=1}$ using $0.65^{\circ} \mathrm{K} / 100 \mathrm{~m}$ lapse rate and $u_{0}, v_{0}$, $q_{0}$ are assumed to be equal to the values at $k=1$.

The vertical diffusion coefficient $K_{v}, K_{H}$ and $K_{q}$ are equal to each other and are assumed to be a simple function of the Richardson number, i.e.,

$$
K_{v}=K_{H}=K_{q}=l^{2} \Psi
$$

where

$$
\Psi=\sqrt{\left(\frac{\partial V}{\partial z}\right)^{2}-\frac{g}{\theta} \frac{\partial \theta}{\partial z}}
$$

Here, $z$ is height and $\theta$ is potential temperature. For the mixing length $l$, constant value of $30 \mathrm{~m}$ is used. Furthermore, we set minimum limits for $K$ as $K_{v}=K_{H}=1 \mathrm{~m}^{2} \mathrm{sec}^{-1}$ and $K_{q}=5 \mathrm{~m}^{2}$ $\sec ^{-1}$. We also assume $K_{v}=K_{H}=1 \mathrm{~m}^{2} \mathrm{sec}^{-1}$ and $K_{q}=5 \mathrm{~m}^{2} \mathrm{sec}^{-1}$ when $\Psi$ is imaginary.

For the surface fluxes, $C_{D}, C_{H}$ and $C_{q}$ are also assumed to be a function of the Richardson number (Kondo, 1975), i.e.,

$$
\begin{aligned}
& C_{D}=C_{D} * \cdot \frac{C_{D}}{C_{D} *} \\
& C_{H}=C q=C_{H} * \cdot \frac{C_{H}}{C_{H} *}
\end{aligned}
$$

here

$$
\frac{C_{D}}{C_{D^{*}}}= \begin{cases}0.1 & S \leqq-3.0 \\ 0.1+0.03 S+0.9 e^{4.3 S} & -3.0<S<0.0 \\ 1.0+0.47 \sqrt{S} & 0.0 \leqq S \leqq 3.0\end{cases}
$$

and

$$
\frac{C_{H}}{C_{H^{*}}}= \begin{cases}0.1 & S \leqq-3.0 \\ 0.1+0.03 S+0.9 e^{4.8 S} & -3.0<S<0.0 \\ 1.0+0.63 \sqrt{S} & 0.0 \leqq S \leqq 3.0\end{cases}
$$

where

$$
\begin{aligned}
& S=S_{0} \frac{\left|S_{0}\right|}{\left|S_{0}\right|+0.01} \\
& S_{0}=\frac{T_{S F}-T_{0}}{\left|\vec{V}_{0}^{2}\right|}
\end{aligned}
$$

Maximum limit of $S_{0}$ is set to 3.0.

$C_{D}{ }^{*}$ and $C_{H}{ }^{*}$ are the drag coefficients for momentum and heat for neutral stratification and are defined as

$$
C_{D} * \begin{cases}=0.0012+0.000025\left|\vec{V}_{0}\right| ; \text { ocean } \\ =0.0043 & ; \text { land } \\ =0.0012 & ; \text { ice }\end{cases}
$$

and

$$
C_{H} * \begin{cases}=0.00128 ; & \text { ocean } \\ =0.0043 ; & \text { land } \\ =0.0012 ; & \text { ice }\end{cases}
$$

In order to prevent numerical instability, fully implicit time integration scheme is applied to those terms.

(c) Cumulus parameterization

Kuo's (1974) parameterization scheme is applied. The conditions for the release of convective heating are;

(i) Conditionally unstable stratification, i.e.,

$$
\frac{\partial \theta_{e}}{\partial p}>0 ; \quad \theta_{e}=\theta \exp \left(\frac{L q_{s}}{C_{p} \dot{T}}\right)
$$

(ii) Positive moisture convergence in the cloud layer, i.e.,

$$
I=\int_{\sigma_{B}}^{\sigma_{T}} \frac{\partial q}{\partial t} d \sigma>0
$$

(iii) Mean relative humidity in the cloud layer exceeding the critical value $\left(r_{c}=90 \%\right)$, i.e.,

$$
\int_{\sigma_{B}}^{\sigma_{T}} q d \sigma / \int_{\sigma_{B}}^{\sigma_{T}} q_{s} d \sigma \geqq r_{c}
$$

where $\theta_{e}$ is the equivalent potential temperature, $L$ is the latent heat of condensation and the subscripts $T$ and $B$ stand for cloud top and base, respectively. The cloud base is assumed to be the first Ievel above the PBL $(k=4)$ where $\partial \theta_{e} / \partial p>0$. Cloud top is defined as the level where the moist adiabat starting from the cloud base intersects the temperature of the model $\left(T_{c}=T\right)$.

The heating and moistening rates are assumed to take the forms,

$$
\begin{aligned}
& \frac{\partial T}{\partial t}=(1-b) I \cdot \frac{T_{c}-T}{\int_{\sigma_{B}}^{\sigma_{T}}\left(T_{c}-T\right) d \sigma} \cdot \frac{L}{C_{p}} \\
& \frac{\partial q}{\partial t}=b I \cdot \frac{q_{s}-q}{\int_{\sigma_{B}}^{\sigma_{T}}\left(q_{s}-q\right) d \sigma}
\end{aligned}
$$

where $q_{s}$ is the saturation specific humidity for temperature $T$.

The partitioning factor ' $b$ ' has been assumed to be a function of mean relative humidity, but following Krishnamurti et al. (1980) $b=0$ is 
presently used.

The computation of the cumulus parameterization is performed at the Gaussian grids and adjustment type scheme is applied. The procedure is as follows:

i) Temperature and moisture are predicted without cumulus heating.

ii) Predicted $T_{n}{ }^{m}$ and $q_{n}{ }^{m}$ are converted to grid point values.

iii) $T$ and $q$ at the grid points are modified according to Kuo's scheme.

iv) Modified $T$ and $q$ are converted from grid to spectral domain.

(d) Radiative process

The radiation scheme similar to that proposed by Katayama (1972) is used for estimating heating rate due to long- and short-wave radiation.

(i) Cooling rate due to long-wave radiation

$$
\begin{aligned}
(d T / d t)_{z} z^{\text {long }}=\left(g / C_{p}\right) \times\left(d F_{z} / d p\right) & \\
F_{z}= & F_{z}^{\uparrow}-F_{z^{\downarrow}} \\
F_{z} \uparrow= & \pi B_{z}+\int_{\pi B_{z}}^{\pi B_{g}} \tau\left(u_{z} *-u^{*}, \bar{T}\right) d(\pi B) \\
F_{z} \downarrow= & \pi B_{z}-\pi B_{c} \tilde{\tau}\left(u_{\infty}^{*}-u_{z}{ }^{*}, T_{c}\right) \\
& -\left(\pi B_{\infty}-\pi B_{c}\right) \tau\left(u_{\infty}^{*}-u_{z}^{*}, \bar{T}\right) \\
& -\int_{\pi B_{\infty}}^{\pi B_{z}} \tau\left(u^{*}-u_{z}^{*}, \bar{T}\right) d(\pi B) \\
\pi B_{z}= & \sigma T_{z^{4}}, \quad \pi B_{c}=\sigma T_{c}^{4}, \quad \pi B_{\infty}=\sigma T_{\infty}^{4}, \\
& T_{c}=220^{\circ} \mathrm{K}, \quad T_{\infty}=180^{\circ} \mathrm{K}
\end{aligned}
$$

where $\sigma$ is the Stefan-Boltzman constant and $\bar{T}=260^{\circ} \mathrm{K} . \quad T_{c}$ is a critical temperature which is used in the derivation of eq. (84) from the original equation (see Katayama, 1972). $u_{z}{ }^{*}$ is the equivalent optical length including cloud effects. Transmissivities ( $\tau$ and $\tilde{\tau}$ ) are estimated by Katayama's empirical formula. Clouds are assumed to be a black body. The vertical resolution is doubled for numerical integration of (83) and (84) in order to better resolve the change of lapse rate near the tropopause.

(ii) Heating rate due to short-wave radiation

$$
(d T / d t)_{z}{ }^{\text {short }}=\left(g / C_{p}\right) \times\left(d S_{z} \downarrow / d p\right)
$$

$S_{z} \downarrow$ is downward radiative flux due to shortwave radiation. It is assumed to depend on water vapor and ozone distribution. A climatic heating rate due to ozone that depends on solar position, latitude and pressure is used in the model stratosphere (uppermost 3 layers). A short-wave heating rate due to water vapor is estimated from the function proposed by Manabe and Möller (1961). (iii) Cloud amount

The cloud amount is assumed to be a function of the predicted relative humidity and sigma. It is assumed that cloud at one level does not overlap with that at other levels. Furthermore, it is assumed that the summation of the maximum cloud amount over all the levels is equal to 1.0. A relationship between relative humidity and cloud amount is as follows;

$$
\begin{gathered}
C L_{k}=C_{k} \cdot(0.025 \times R H-1.5) \\
\quad \text { for low cloud }(K=4) \\
C L_{k}=C_{k} \cdot(0.0154 \times R H-0.54) \\
\quad \text { for middle cloud }(K=5,6,7) \\
C L_{k}=C_{k} \cdot(0.0066 \times R H-0.16) \\
\quad \text { for high cloud }(K=8,9) \\
\sum_{K=4}^{9} C_{k}=1.0
\end{gathered}
$$

$R H$ is relative humidity (\%). $C L_{K}$ and $C_{K}$ are the cloud amount and a weighting parameter for the three types of cloud (high, middle and low).

The radiation is calculated every three hours. It is assumed that heating rate due to radiation is constant during the three hours.

(e) Prediction of ground surface temperature

The ground surface temperature $\left(T_{g}\right)$ is predicted using the following equations.

$$
\begin{aligned}
& \frac{\partial T_{g}}{\partial t}=-C_{g} \cdot H_{a} \\
& H_{a}=\sigma_{B} T_{g}^{4}+S_{h} \uparrow+E^{\uparrow}-\delta_{f} \cdot S \downarrow \cdot\left(1-\alpha_{s}\right)-R \downarrow
\end{aligned}
$$

where

$C_{g}$ : Constant dependent on the condition of the ground surface (ice or bare land)

$\sigma_{B}:$ Stefan-Boltzman constant

$S_{h}^{\uparrow}$ : Sensible heat flux from the ground surface

$E^{\uparrow}$ : Evaporation from the ground surface

$S \downarrow$ : Solar radiative flux at the ground

$R^{\uparrow}$ : Long-wave radiative flux at the ground $\alpha_{s}:$ Albedo of the ground

$\delta_{f} S \downarrow$ is the effective incoming solar radiation flux. $\delta_{f}$ is an empirical parameter determined by surface soil conditions. At the initial time, $T_{g}$ is computed assuming $H_{a}=0 . \quad S \downarrow$ and $R \downarrow$ are assumed to be constant during the three hours. For the time differencing, fully implicit scheme is applied. 


\section{(f) Topography}

$1 \times 1$ degree latitude/longitude tabulation of topography has been converted to the spherical harmonics truncated at wavenumber 42 . The waves are modified to smooth out small scale features using the following exponential type filter (Hoskins, 1980).

$$
\left(\Phi_{s_{n}}^{m}\right)_{\text {smoothed }}=\Phi_{s_{n}}^{m} \exp \left(-k n^{2}(n+1)^{2}\right)
$$

For the parameter $k, 0.7 \times 10^{-6}$ is used.

(g) Other climatologically specified parameters

Monthly climatologies of albedo, sea surface temperature, ground wetness and ice cover are used for planetary boundary layer and the radiation calculation.

\section{Initialization}

(a) Pressure to sigma and sigma to pressure conversion

Cubic spline interpolation is applied to the vertical interpolation of winds. Temperature at the sigma levels are obtained from the vertical derivatives of the heights fitted to the cubic spline. Simple linear interpolation is used for dew point depressions.

(b) Nonlinear normal mode initialization

The nonlinear normal mode initialization procedure proposed by Machenhauer (1977) is applied to suppress the gravity wave noise. The principle of the scheme is to set time tendencies of the gravity modes in the initial field to zero.

The normal modes used to separate gravity modes from initial data are computed for isothermal $\left(\bar{T}=273^{\circ} \mathrm{K}\right)$ atmosphere at rest. Vertical discretization of the atmosphere is made according to the model sigma levels. The vertical modes up to 5 and modes of period less than 48 hours are initialized, i.e., their time tendencies are set to zero.

The prediction model excluding latent heating and boundary layer processes is used to calculate the tendencies. The number of iterations for the initialization procedure is set to 4 . Slight modification to the Machenhauer's iteration procedure is made for iteration count 3 and 4 in order to assure the convergence of the iteration scheme.

\section{Analysis methods}

Details of the analysis scheme is described in the annual report of the Staff Members, ECC, JMA (1983) and only brief description will be made here.
Analysis methods are different in the troposphere (below $100 \mathrm{hPa}$ ) and in the stratosphere. In the troposphere, two dimensional multi-variate statistical interpolation method is used for geopotential and winds. Water vapor (dew-point depression) is analyzed using uni-variate interpolation. For the guess field, 12 hour forecast is used. In the stratosphere, 2-dimensional functional fitting method is used. For the base functions, sinusoidal functions in east-west, and $B$ spline functions in latitude are used. Winds and heights are analyzed simultaneously assuming geostrophic relation north of $30^{\circ} \mathrm{N}$.

\section{Comparison of forecast with the operational grid point model}

The description of the operational grid point model is found in the Staff Members, ECC (1980). The model has 8-levels in the vertical and $381 \mathrm{~km}$ (at $60^{\circ} \mathrm{N}$ ) grid over the stereographic projection. The model includes latent heating, convective adjustment, surface exchange processes but does not incorporate radiation and soil-air exchange processes. It should be also noted that the horizontal resolution of the grid point model is somewhat coarser than that of the spectral model which roughly corresponds to $270 \mathrm{~km}$ grids. Therefore, the comparison of the two models should not be regarded as a simple comparison of grid point vs. spectral methods.

Figure 2 shows comparison of monthly averaged tendency correlations of height at $500 \mathrm{hPa}$ over the northern hemisphere (north of $20^{\circ} \mathrm{N}$ ). Solid line represents correlations for the spectral model and dashed lines for the grid point model. The 72 hour forecast by the spectral model is nearly equal or slightly better than the 48 hour forecast of the grid point model. The mean error (monthly averaged forecast error) is also much smaller in the spectral model as shown in Fig. 3.

Besides the objective scores, subjective evaluation of the both models also indicated that the spectral model is superior in the following respects.

(1) Phase error of the disturbances are much small. This is an essential improvement particularly for real data forecasting. Recent statistics of the phase speed of the disturbances in the spectral model indicate still underestimation but the magnitude is much less than the grid point model. Since there is no phase error associated with the finite differencing in the spectral model, this phase error should be related to physical 
and dynamical reasons.

(2) The tendency of the disturbance to weaken with time was much reduced in the spectral model. This is also considered to be due to the use of the spectral method as well as to the better resolution.

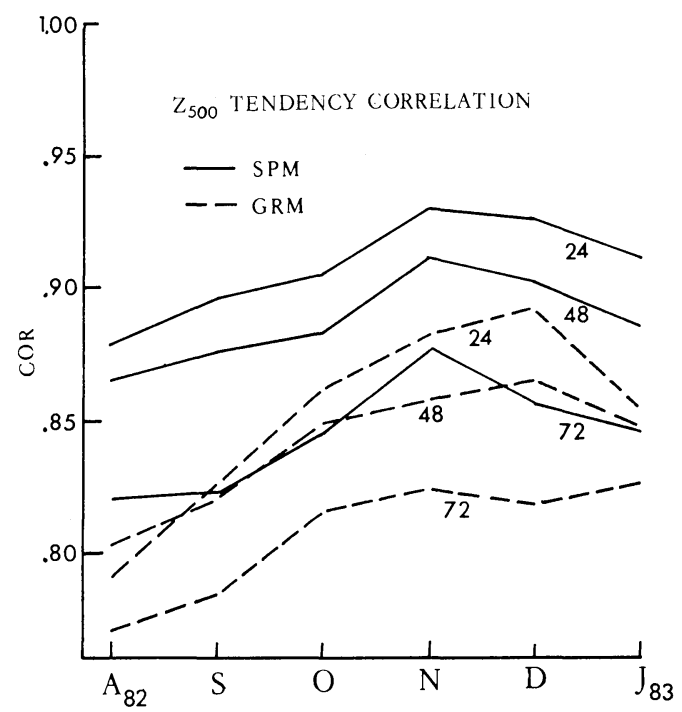

Fig. 2 Comparison of monthly averaged 500 $\mathrm{hPa}$ height forecast tendency correlations for the spectral (solid ilnes) and the grid point (dashed lines) models averaged over the northern hemisphere (north of $20^{\circ} \mathrm{N}$ ). The correlations for August 1982 to January 1983 are shown. Numbers attached to the correlation lines are the forecast hours.

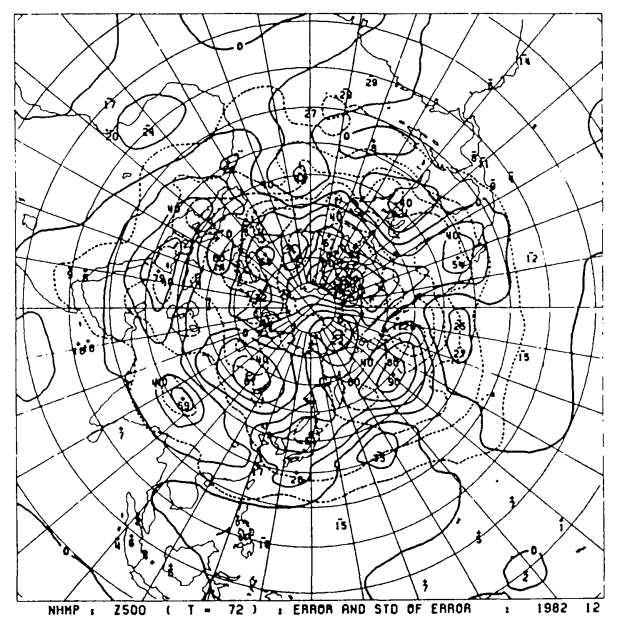

\section{Performance of the spectral model}

The spectral model has been run twice a day for 72 hour forecast. In addition, 192 hour (8 day) forecast has been made twice a week. Forecast statistics taken during spring 1983 will be presented in this section.

Table 2 is the monthly averaged tendency correlation of various parameters computd over the northern hemisphere (north of $20^{\circ} \mathrm{N}$ ), for 24 , 48 and 72 hour forecasts. It is clear from this table that the forecast is best for $500 \mathrm{hPa}$ height. At other levels, the forecast accuracy drops down. Poor forecast at $50 \mathrm{hPa}$ is due to the

Table 2 Forecast tendency correlation of various variables averaged for March 1983 over the northern hemisphere (north of $20^{\circ} \mathrm{N}$ ).

\begin{tabular}{cccc}
\hline Forecast time & $24 \mathrm{hr}$ & $48 \mathrm{hr}$ & $72 \mathrm{hr}$ \\
\hline Ps & .87 & .86 & .82 \\
Z700 & .90 & .88 & .83 \\
Z500 & .93 & .90 & .85 \\
Z200 & .91 & .89 & .85 \\
Z050 & .67 & .71 & .70 \\
T850 & .78 & .80 & .77 \\
T500 & .84 & .84 & .80 \\
T300 & .60 & .65 & .65 \\
T050 & .37 & .51 & .54 \\
V850 & .78 & .77 & .73 \\
V500 & .83 & .82 & .79 \\
V200 & .82 & .82 & .79 \\
V050 & .43 & .55 & .56 \\
\hline \multicolumn{5}{c}{ March 1983} &
\end{tabular}

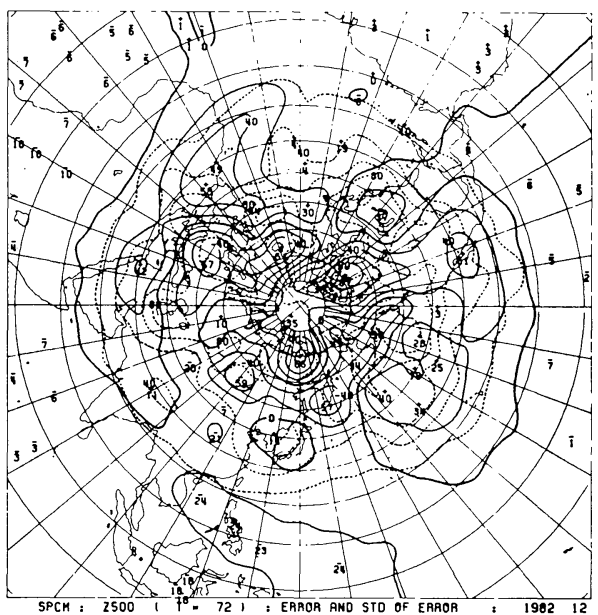

Fig. 3 Comparison of monthly mean December (1982) $500 \mathrm{hPa}$ height error for the grid point model (left) and for the spectral model (right). Standard deviation of error is shown by dashed lines. Contour interval is $20 \mathrm{~m}$ for geopotential and $10 \mathrm{~m}$ for standard deviations. 

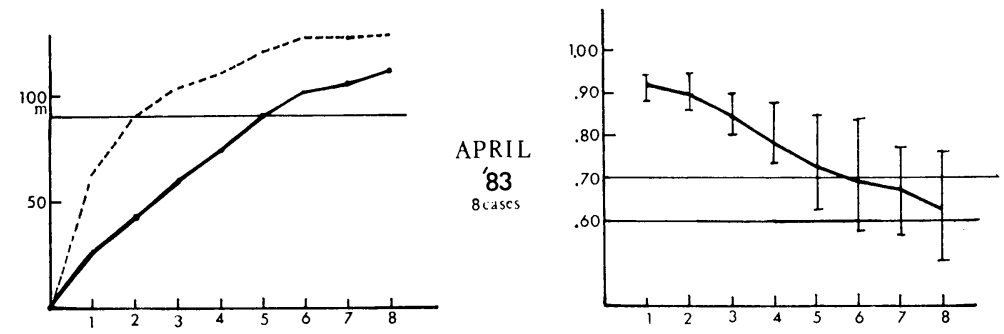

$Z_{500}$ RMS ERROR

$20 N-90 N$
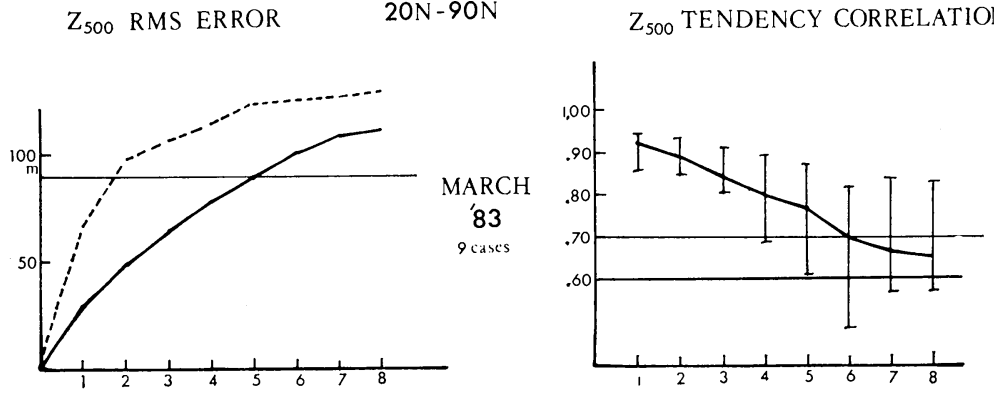

Fig. 4 Variation of the $500 \mathrm{hPa}$ root mean square error (left) and tendency correlations (right) with prediction time for the spectral model averaged over the northern hemisphere (north of $20^{\circ} \mathrm{N}$ ) 9 cases from March 1983 (bottom) and 8 cases from April 1983 (top). For the RMS, persistency is shown by the dashed lines. Thin horizontal lines are the estimated RMS of climatology (RMS at day 8 devided by $\sqrt{2}$ ). For the tendency correlations, upper and lower limit of the cases are presented by thin lines.

fictitious north-south gradient of height resulted from combined effect of vertical finite differencing scheme and the insufficient vertical resolution at the tropopause level. This fictitious gradient tends to be reduced as forecast proceeds, and the forecast score improves. For other variables, temperature is predicted fairly well at 850 and $500 \mathrm{hPa}$. Inferior forecast at $300 \mathrm{hPa}$ is again due to the model resolution in the vertical which does not sufficiently resolve the change of lapse rate at the tropopause. Wind forecast is reasonably good except in the stratosphere.

The performance of the spectral model in the medium range forecast is presented in Fig. 4. Averages of 8 cases during April and 9 cases during March 1983 show that tendency correlation of $500 \mathrm{hPa}$ height (right side figures) stays above 0.6 level, which is considered to be the useful limit for forecast (Hollingsworth et al., 1980). Upper and lower limit of individual case presented by the thin lines show that correlation stays over 0.8 level after 8 days in some cases. The root mean square errors of $500 \mathrm{hPa}$ height are shown in the left side of
Fig. 4. If we set the predictability limit as a point where forecast RMS curve intersects RMS of climatology, the limit of predictability is about 5 days. In the Figure, RMS of climatology shown by thin lines are determined by dividing RMS of persistence at day 8 by square root of $2 . *$

\section{Some synoptic examples of the forecast}

(1) Sudden development of a baroclinic disturbance over Japan (March 16-17, 1983)

In the spring time, sudden bursting development of baroclinic disturbances are frequently cobserved over Japan and nearby oceans. These disturbances are similar to the spring storms in the east coast of U.S. Recently, Bengtsson (1983) conducted detailed prediction experiments on such bursting baroclinic development. The aim of the present paper is not to go into the detail of the forecast but just to show the ability of the present model which can forecast such

* This relation of RMS between the climatology and the persistency can be easily derived if one assumes infinite number of forecast samples. 
developments 3 days in advance.

On the left side of Fig. 5, observed fields of surface pressure are shown. A weak low located over the central China $\left(40^{\circ} \mathrm{N}, 105^{\circ} \mathrm{E}\right)$ moves southeastwards with little development until March 16 and reaches over the Korean peninsula. At this time, another weak low formed about 8 degrees south-southeast of the low. During the next 24 hours, two lows moved along the northern and southern coasts of Japan and merged at the Pacific coast to the north of the central Japan to become an intense low as shown in the left bottom figure.

The forecast presented in the right side of Fig. 5, shows that the model is capable of predicting such sudden development with considerable accuracy. Similar such successful forecasts are encountered during the spring period 1983. Comparing the present forecast with the previous operational grid point model, removal of dissipative tendency by the use of spectral method and increased resolution seem to be responsible for the good performance of the spectral model.

(2) Forecast of Typhoon (Aug. 27, 1982.

\section{Typhoon ELLIS)}

Prediction of Typhoon position and intensity are one of the most essential requirements at JMA. In the operational system, typhoon in the initial field is analyzed by the bogus data, which is automatically generated from typhoon location, central pressure and its radius using climatological typhoon structures. This procedure is essential in analyzing typhoon from very little observations and in maintaining typhoon circulation in the large scale model.

The example presented here is one of the most successful cases of the forecast of typhoon movement and intensity changes. As shown in Fig. 6, northward movement and intensity changes during the 3 days are extremely well predicted. Considering the coarseness of the horizontal resolution of the model, the success of the forecast is surprising. Bengtsson et al. (1983) showed that ECMWF model of somewhat finer resolution $\left(1.875^{\circ}\right.$ lat/lon) generates typhoons and hurricanes during the 10 day forecast period but correspondence with real atmosphere were not particularly good. The present case seems to indicate that if one can provide proper typhoon structures in the initial field, even somewhat coarser resolution model can still predict tropical cyclones.

The maintenance of the vortex is much inferior in the coarse mesh grid point model (it rarely maintains typhoon circulation for 24 hours). We feel that the spectral method that ensures accurate horizontal advection (Gottlieb and Orszag, 1977) contributes to the maintenance of typhoon circulation in the spectral model.

For other cases, typhoon circulation does not last for three days. It indicates that pure advection is not sufficient for the maintenance of the circulation and some other mechanisms (e.g. cumulus heating) are essential. Since even the mechanisms of maintenance of the typhoon in the spectral model is not clear at the moment, we have just started basic investigation to look into the maintenance problem which hopefully leads to the better forecast of typhoon by the model.

(3) An example of 8-day forecast (Initial state, April 27 12Z, 1983)

Due to the operational requirement of issueing one week forecast, 8-day integration of the spectral model has been performed twice a week (Sunday and Wednesday, 12 GMT). In order to make medium range forecast of the time scale of 5-10 days, global model is preferable. However, due to the limitation on the computer, we are currently applying hemispheric model for the 8-day forecast. We have considered possibility of expanding the model to global by decreasing the number of vertical levels. However, we have rejected the idea since high resolution in the vertical for hemispheric model gives better 3-day forecast than the global model of less vertical resolution. It is an interesting problem to find optimum vertical/ horizontal resolution with global/hemispheric domain.

The example chosen here is a case which has assisted the forecasters in predicting fine weather few days later. The forecast at day 4 and 8 (upper panels) and the corresponding verifying analyses (lower panels) are shown in Fig 7. From forecaster's point of view, the location of trough in relation to the Japanese islands are of interest, which is fairly well predicted even after 8 days. The formation of blocking to the far north of Japan on day 8 is also reasonably well predicted. As shown in the bottom of the upper right panel, tendency correlation (TCOR) of $500 \mathrm{hPa}$ height on day 8 is 0.7 .

\section{Conclusions}

A hemispheric spectral model has been developed for operational purpose. The model has 12 levels in the vertical and horizontal resolution 

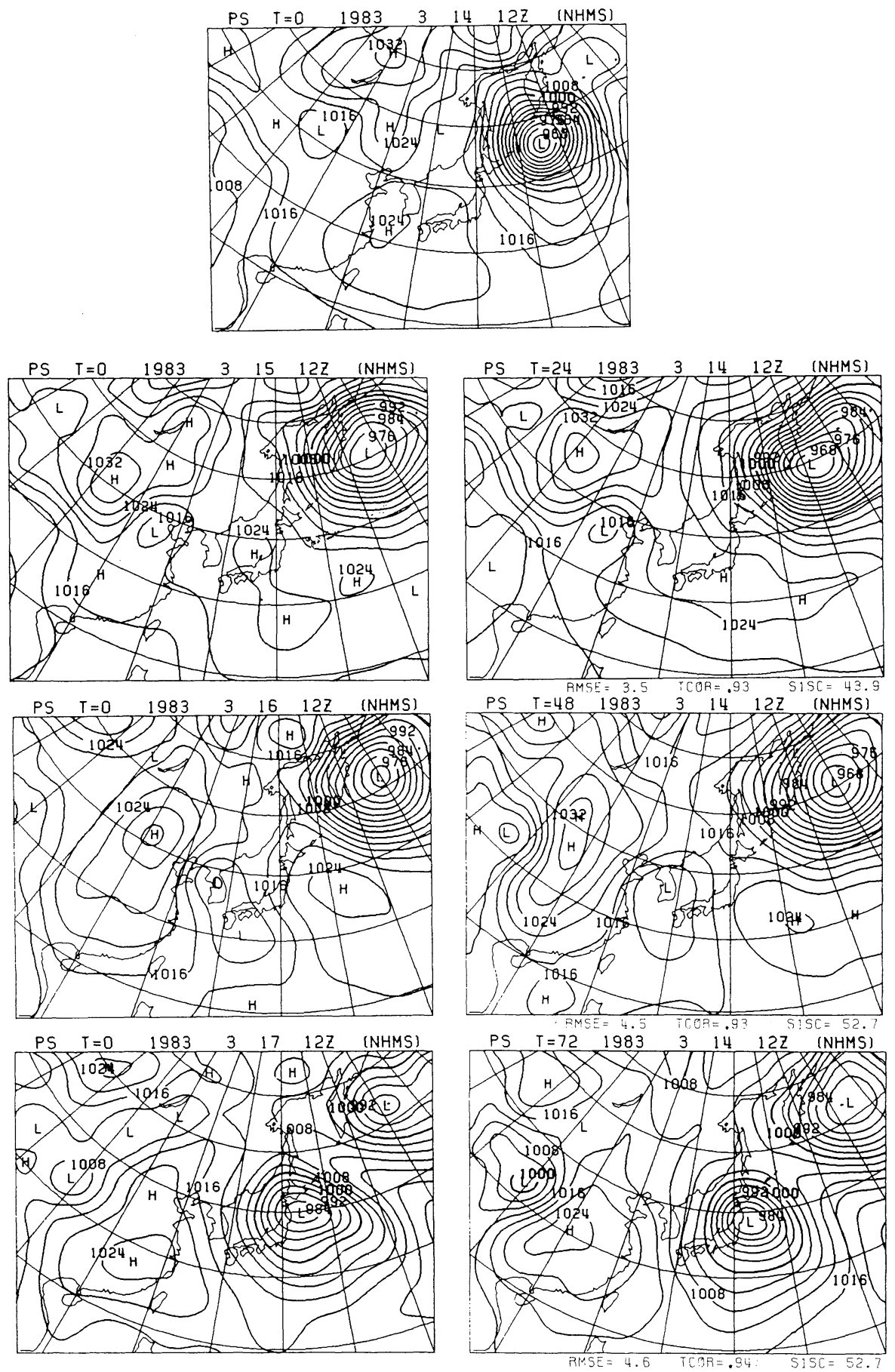

Fig. 5 An example of a prediction of an intense low. Top panel is initial field. Left 3 panels are observation and right 3 panels are the forecasts. The data and time shown on the right panels are the initial time. $\mathrm{T}$ represents forecast hour. Contour interval $4 \mathrm{hPa}$. 

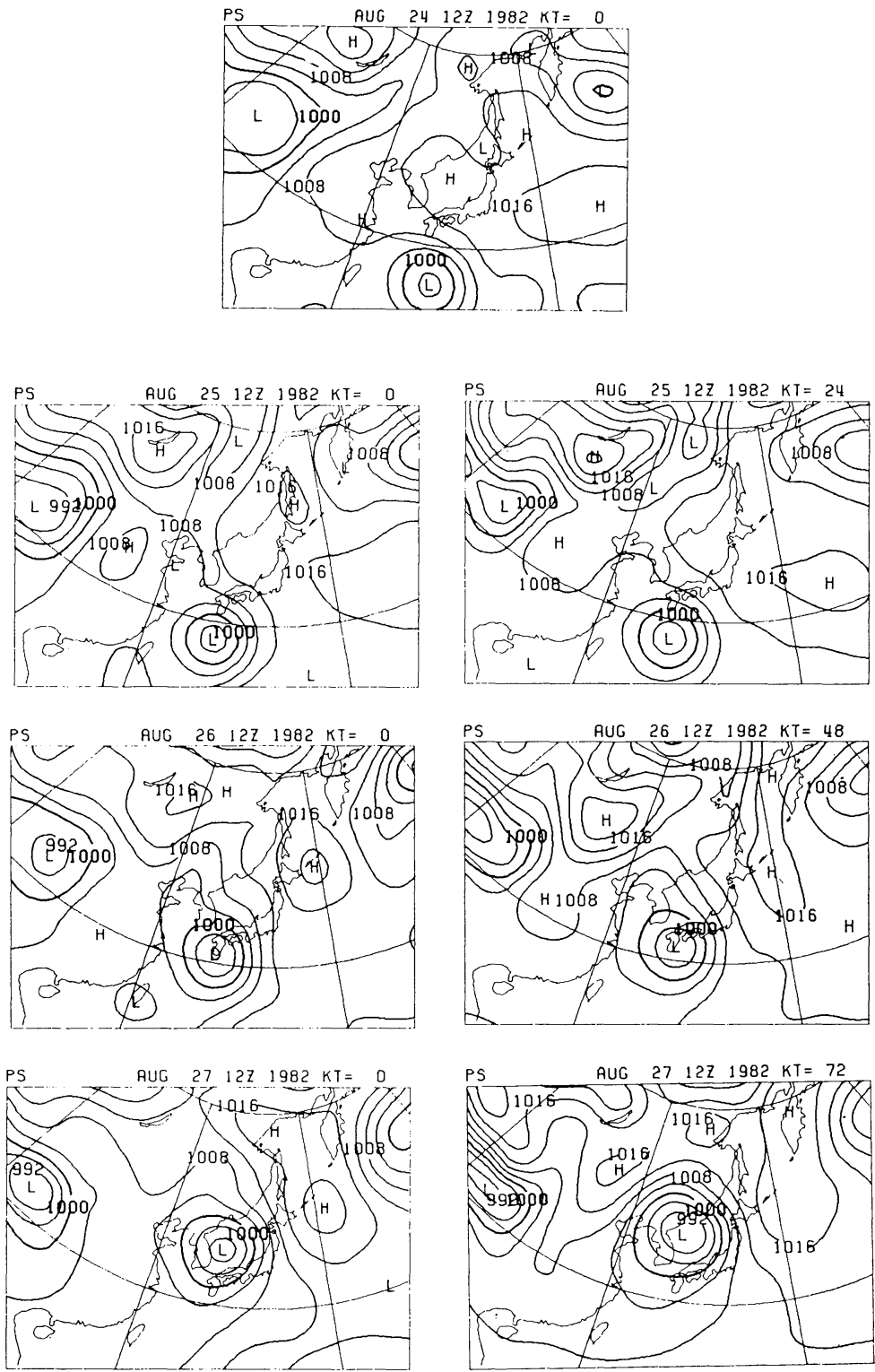

Fig. 6 An example of a forecast of the typhoon ELLIS.

of triangular 42 truncation. The model incorporates latent heating, convective parameterization, surface exchange processes, vertical diffusion, radiation with effects of clouds and diurnal variation and ground soil surface processes. Other components of the forecast-analysis system includes multi-variate optimum interpolation analysis and nonlinear normal mode initialization.

The model has been tested daily for nearly a year and compared with the operational grid point model. The subjective and objective evaluation of the models indicate that the spectral model is superior to the grid point model.

The prediction by the spectral model is found to be useful for at least up to 5 to 6 days. The model predicts phase speeds and amplitudes of the disturbances with reasonable accuracy. It has also been shown that the model is capable of predicting intense development of baroclinic disturbances 3 days in advance. The model also predicts typhoon movements with some accuracy. 

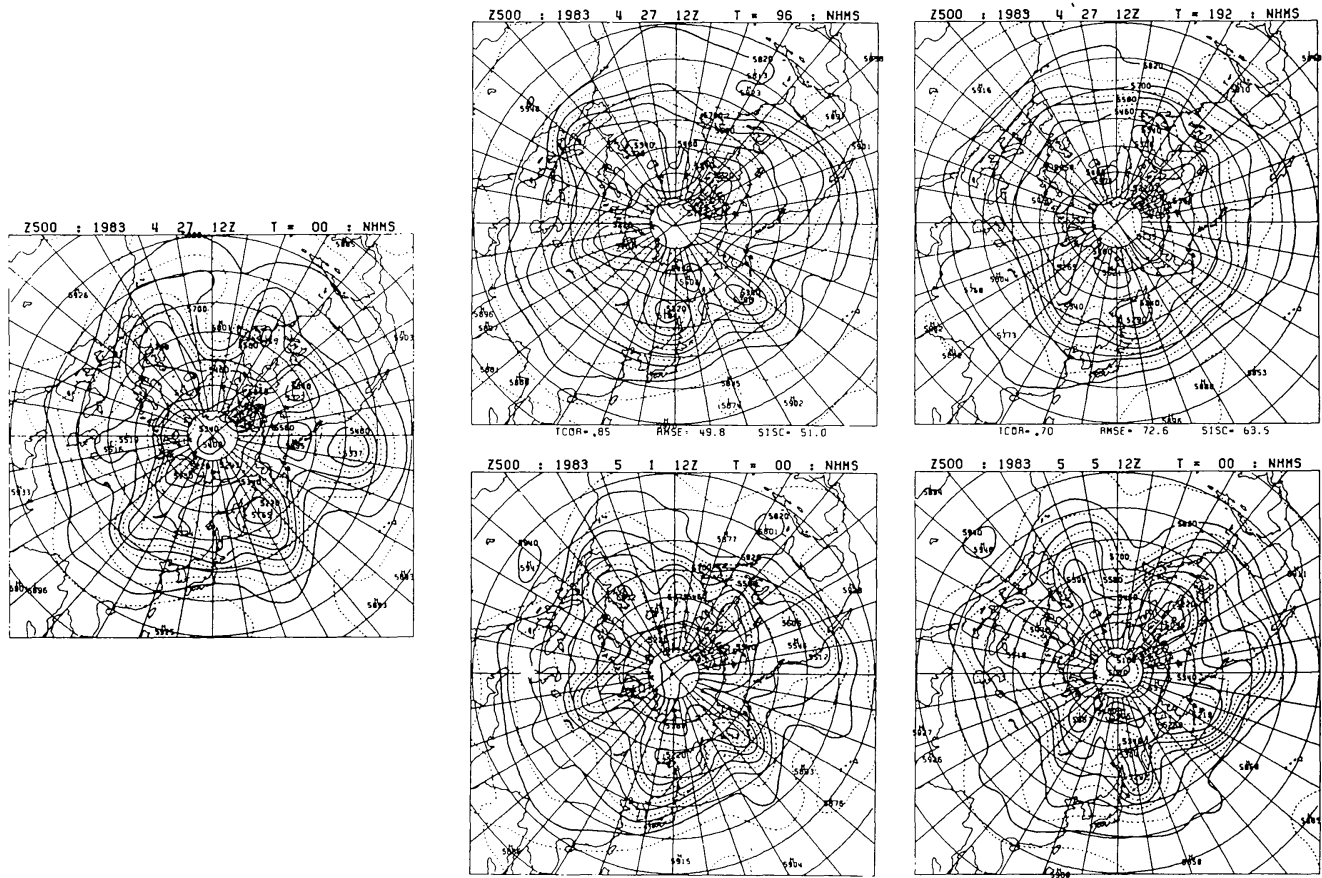

Fig. 7 An example of 8-day $500 \mathrm{hPa}$ height forecast. Initial field (left), 4 day forecast (middle) and 8 day forecast (right) are shown at the top. Corresponding verifying analyses are shown at the bottom. Contour interval is $60 \mathrm{~m}$.

The good performance of the spectral model may be explained by a number of reasons, and are listed below.

(1) Higher resolution both in the vertical and in the horizontal.

(2) Better physical processes.

(3) Spectral method that eliminates computational deficiencies due to finite differencing.

(4) Nonlinear normal mode initialization that does not require any unnatural treatment at the low latitudes.

(5) Multi-variate optimum interpolation analysis scheme that minimize imbalance between the mass and the motion field.

(6) Vertical extent of the model resolving stratosphere, together with the stratospheric analysis scheme.

(7) Other improvements in data handlings, e.g., data checking, decoding, etc.

One other important difference (not an improvement) should be noted in relation to the normal mode initialization. The initialization method allows us to use mass and motion informations at the same time for the initial field. However, the Machenhauer's iteration scheme places more weight to the motion field than to the mass field. Therefore, in the spectral model, wind observations is the major input for the initial state. On the contrary, in the grid point model system, we have been using balance equation approach, which uses only the mass field information. The difference seems to be important and its effects needs further researches.

It is important to realize here that forecast model is just a part of the entire analysis-forecast system. Good forecast cannot be made unless all the parts in the system are of good and balanced quality. Furthermore, we should consider that the parts in the system may interact and feed the products back. For example, better analysis produces better forecast, which feeds back better guess for the analysis. In such a system, it is probably meaningless to look for one factor that contributes mostly to the performance of the entire system. Thus, we would like to make conclusion that the better forecast produced by the spectral model is due not just to the use of spectral method but to the improvements in entire analysis-forecast system.

In order to improve the forecast further, two approaches are possible. One approach is to 
grade up the system, i.e. increase of horizontal and vertical resolution and domain, improvement of physical processes, incorporation of 3-dimensional optimum interpolation, etc. However, we are still not sure whether such increase of sophistication in the model and analysis can really increase the forecast accuracy. Numerous forecasts with such sophisticated system may provide some answer to the question. The other approach is to analyze the forecast error from various point of view, and to find the cause of the error. This subject will be of considerable interest and be elaborated in the papers to follow.

\section{Acknowledgements}

We would like to thank Messrs K. Kashiwagi, M. Sugi, T. Nakayama, Dr. T. Hiraki and other members of the Electronic Computation Center of JMA for their efforts and cooperations in organizing forecast-analysis system. We are also grateful to Drs. T. Yoshida, Ta. Nitta and $\mathrm{K}$. Ninomiya for their continuous encouragements throughout the work.

\section{Appendix I}

The basic equations in the physical space are as follows:

\section{Vorticity equation}

$$
\dot{\zeta}=E+\mathscr{D}_{\zeta}
$$

where

$$
\begin{aligned}
E= & -\frac{1}{a \cos ^{2} \phi}\left(\frac{\partial A}{\partial \lambda}+\cos \phi \frac{\partial B}{\partial \phi}\right) \\
A= & (\zeta+f) U+\dot{\sigma} \frac{\partial V}{\partial \sigma}+\frac{R T^{\prime}}{a} \cos \phi \frac{\partial \pi}{\partial \phi} \\
& +\cos \phi \frac{g}{p_{s}} \frac{\partial \tau_{\phi}}{\partial \sigma} \\
B= & (\zeta+f) V-\dot{\sigma} \frac{\partial U}{\partial \sigma}-\frac{R T^{\prime}}{a} \frac{\partial \pi}{\partial \lambda}-\cos \phi \frac{g}{p_{s}} \frac{\partial \tau_{\lambda}}{\partial \sigma} \\
\mathscr{D}_{\zeta}= & K\left(\nabla^{4} \zeta+\frac{4 \zeta}{a^{4}}\right)
\end{aligned}
$$

Divergence equation

$$
\begin{aligned}
& \dot{D}=F+G+\mathscr{D}_{D} \\
& F=\frac{1}{a \cos ^{2} \phi}\left(\frac{\partial B}{\partial \lambda}-\cos \phi \frac{\partial A}{\partial \phi}\right)-\nabla^{2}\left(\frac{U^{2}+V^{2}}{2 \cos ^{2} \phi}\right) \\
& G=-\nabla^{2}(\Phi+R \bar{T} \pi)
\end{aligned}
$$

Other important relations are:

$$
\zeta=\nabla^{2} \phi
$$

$$
\begin{aligned}
& D=\nabla^{2} \chi \\
& U=u \cos \phi=-\frac{\cos \phi}{a} \frac{\partial \phi}{\partial \phi}+\frac{1}{a} \frac{\partial \chi}{\partial \lambda} \\
& V=v \cos \phi=\frac{1}{a} \frac{\partial \phi}{\partial \lambda}+\frac{\cos \phi}{a} \frac{\partial \chi}{\partial \phi} \\
& \pi=\ln p_{s} \\
& T^{\prime}=T-\bar{T} \\
& \bar{T}=\text { constant }=300^{\circ} \mathrm{K} \\
& \mathscr{D}_{D}=K\left(\nabla^{4} D+\frac{4 D}{a^{4}}\right)
\end{aligned}
$$

Thermodynamic equation

$$
\begin{aligned}
\dot{T}= & -\frac{1}{a \cos ^{2} \phi}\left(\frac{\partial U T^{\prime}}{\partial \lambda}+\cos \phi \frac{\partial V T^{\prime}}{\partial \phi}\right) \\
& +T^{\prime} D-\dot{\sigma} \sigma^{\kappa} \frac{\partial T \sigma^{-\kappa}}{\partial \sigma}+\frac{R T}{C_{p}}\left(\frac{\partial \pi}{\partial t}+V \cdot \nabla \pi\right) \\
& +\frac{Q}{C_{p}}+\frac{1}{C_{p}} \frac{g}{p_{s}} \frac{\partial F_{H}}{\partial \sigma}+\mathscr{D}_{H}=H+J+\mathscr{D}_{H}
\end{aligned}
$$

where

$$
\begin{aligned}
H= & -\frac{1}{a \cos ^{2} \phi}\left(\frac{\partial U T^{\prime}}{\partial \lambda}+\cos \phi \frac{\partial V T^{\prime}}{\partial \phi}\right)+L \\
L= & -\dot{\sigma}_{A} \sigma^{\kappa} \frac{\partial}{\partial \sigma} T \sigma^{-\kappa}-\dot{\sigma}_{D} \sigma^{\kappa} \frac{\partial}{\partial \sigma} T^{\prime} D \\
& +\frac{R T^{\prime}}{C_{p}} \frac{\partial \pi_{D}}{\partial t}+\frac{R T}{C_{p}}\left(\frac{\partial \pi_{A}}{\partial t}+\vec{V} \cdot \nabla \pi\right) \\
& +\frac{Q}{C_{p}}+\frac{1}{C_{p}} \frac{g}{p_{s}} \frac{\partial F_{H}}{\partial \sigma} \\
J= & -\dot{\sigma}_{D} \sigma^{\kappa} \bar{T} \frac{\partial}{\partial \sigma} \sigma^{-\kappa}+\frac{R \bar{T}}{C_{p}} \frac{\partial \pi_{D}}{\partial t} \\
\vec{V} \cdot \nabla \pi & =\frac{1}{a \cos ^{2} \phi}\left(U \frac{\partial \pi}{\partial \lambda}+V \cos \phi \frac{\partial \pi}{\partial \phi}\right) \\
\mathscr{D}_{H} & =K \nabla^{4} T
\end{aligned}
$$

Moisture equation

$$
\begin{aligned}
& q=M+\mathscr{D}_{q} \\
& M=-\frac{1}{a \cos ^{2} \phi}\left(\frac{\partial U q}{\partial \lambda}+\cos \phi \frac{\partial V q}{\partial \phi}\right)+N \\
& N=-\dot{\sigma} \frac{\partial q}{\partial \sigma}+q D-P+\frac{g}{p_{s}} \frac{\partial F_{q}}{\partial \sigma} \\
& \mathscr{D}_{q}=K \nabla^{4} q
\end{aligned}
$$

where $K$ is the horizontal diffusion coefficient and $a$ is the radius of the earth.

Continuity equation, tendency equation and equation for $\dot{\sigma}$

$$
\begin{aligned}
& \dot{\pi}=\frac{\partial \pi_{A}}{\partial t}+\frac{\partial \pi_{D}}{\partial t} \\
& \frac{\partial \pi_{D}}{\partial t}=-\int_{0}^{1} D d \sigma
\end{aligned}
$$




$$
\begin{aligned}
& \frac{\partial \pi_{A}}{\partial t}=-\int_{0}^{1} \vec{V} \cdot \nabla \pi d \sigma=Z \\
& \dot{\sigma}=\dot{\sigma}_{A}+\dot{\sigma}_{D} \\
& \left(\dot{\sigma}_{D}\right)_{\sigma+\Delta \sigma}=\left(\dot{\sigma}_{D}\right)_{\sigma}-\int_{\sigma}^{\sigma+\Delta \sigma} D d \sigma+\Delta \sigma \int_{0}^{1} D d \sigma \\
& \left(\dot{\sigma}_{A}\right)_{\sigma+\Delta \sigma}=\left(\dot{\sigma}_{A}\right)-\int_{\sigma}^{\sigma+\Delta \sigma} \vec{V} \cdot \nabla \pi d \sigma+\Delta \sigma \int_{0}^{1} \vec{V} \cdot \nabla \pi d \sigma
\end{aligned}
$$

Hydrostatic equation

$$
\frac{\partial \Phi}{\partial \ln \sigma}=-R T
$$

For energy conserving finite differencing scheme,

$$
\frac{\partial \Phi}{\partial \sigma^{\kappa}}=-C_{p} T \sigma^{-\kappa}
$$

is also used.

\section{References}

Arakawa, A., 1972: Design of the UCLA general circulation model. Tech. Rept., No. 7, Dept. of Meteorology, UCLA, CA.

Asselin, R., 1972: Frequency filter for time integrations. Mon. Wea. Rev., 100, 487-490.

Bengtsson, L., 1981: The weather forecast. Pure and Applied Geophysics, 119, 515-537.

H. Böttger and M. Kanamitsu, 1982: Simulation of hurricane-type vortices in a general circulation model. Tellus, 34, 440-457.

- and A. Lange, 1982: Results of the WMO/CAS Numerical Weather Prediction Data Study and Intercomparison Project for forecast for the Northern Hemisphere in 1979-80. World Meteorological Organization, Geneve.

Bourke, W., 1974: A multi-level spectral model. I. Formulation and hemispheric integration. Mon. Wea. Rev., 102, 687-701.

ECMWF, 1982: Seminar 1981. Problems and prospects in long and medium range weather forecasting. ECMWF, Shinfield Park, Reading, U.K.

Eliasen, E., Machenhauer, B., and Rasmussen, E., 1970: On a numerical method for integration of the hydrodynamical equations with a spectral representation of the horizontal fields. Rept., No. 2. Institut for teoretisk Meteorologi, Köbenhavns Universitet, Denmark.

Gottlieb, D. and S. A. Orszag, 1977: Numerical analysis of spectral methods. Theory and Ap- plications. Society for Industrial and applied Mathematics, Philadelphia, Penn., 19103, U.S.A.

Hollingsworth, A., K. Arpe, M. Tiedtke, M. Capaldo and H. Savijarvi, 1980: The performance of a medium-range forecast model in winter-Impact of physical parameterizations. Mon. Wea. Rev., 108, 1736-1773.

Hoskins, B. J., 1980: Representation of the earth topography using spectral harmonics. Mon. Wea. Rev., 108, 11-115.

— , and A. J. Simmons, 1975: A multi-layer spectral model and the semi-implicit method. Quart. J. Roy. Meteor. Soc., 101, 637-655.

Katayama, A., 1972: A simplified scheme for computing radiative transfer in the troposphere. Numerical Simulation of Weather and Climate. Tech. Rept., No. 6, UCLA.

Kondo, J., 1975: Air-sea bulk transfer coefficients in diabatic conditions. Boundary Meteorology, 9, 91-112.

Krishnamurti, T. N., Y. Ramanathan, Hua-lu Pan, R. J. Pasch and J. Molinari, 1980: Cumulus parameterization and rainfall rates I. Mon. Wea. Rev., 108, 465-472.

Kuo, H. L., 1974: Further studies of the parameterization of the influence of cumulus convection on large-scale flow. J. Atmos. Sci., 31, 12321240.

Lorenc, A. C., 1981: A global three-dimensional multivariate statistical interpolation scheme. Mon. Wea. Rev., 109, 701-721.

Machenhauer, B., 1977: On the dynamics of gravity oscillations in a shallow water model with applications to normal mode initialization. Beiträge zur Physik der Atmosphäre, 50, 253-271.

Manabe, S. and F. Möller, 1961: On the radiative equilibrium and heat balance of the atmosphere. Mon. Wea. Rev., 89, 503-532.

Orszag, S. A., 1974: Fourier Series on Spheres. Mon. Wea. Rev., 102, 56-75.

Staff Members, E.C.C., 1980: Outline of operational numerical weather prediction at Japan Meteorological Agency, Periodic report on Numerical Weather Prediction, E.C.C., J.M.A. Available form, E.C.C. at J.M.A.

, 1983: Outline of operational numerical weather prediction at Japan Meteorological Agency, Periodic Report on Numerical Weather Prediction, E.C.C., J.M.A. Available form, E.C.C. at J.M.A. 


\section{JMA・スペクトルモテルについて}

\section{金光正郎・多田一正・工藤達雄・佐藤信夫・伊佐真好}

気象庁電子計算室

毎日の予報を目的とした12層半球スペクトルモデルを開発した。モデルの水平解像力は波数42の三角形切断で あり, 物理過程としては短波放射の日変化や雲の効果等をも含むほぼ完全なものである。

このモデルはルーチンモデルとして今年三月に採用されるまでに約一年間テスト使用され，当時のルーチンモ デルである格子点モデルと比較された。この結果スペクトルモデルは色々な面で格子点モデルょりる優れている ことが分った。この理由はスペクトル法の使用のみによるのではなく, 解像度やイニシャリゼーション, 解析な どによるものである。

このモデルの有用な予報限界は5〜6日である。モデルは中緯度傾圧波の急激な発達や台風の移動などをかなり の精度で予報することがでさる。 